Portland State University

PDXScholar

$1-1-2011$

\title{
Quantitative Carré Differential Interference Contrast Microscopy to Assess Phase and Amplitude
}

Donald D. Duncan

Portland State University

David G. Fischer

Amanda Dayton

Scott A. Prahl

Follow this and additional works at: https://pdxscholar.library.pdx.edu/ece_fac

Part of the Electrical and Computer Engineering Commons Let us know how access to this document benefits you.

\section{Citation Details}

Duncan, D. D., Fischer, D. G., Dayton, A., \& Prahl, S. A. (2011). Quantitative Carre differential interference contrast microscopy to assess phase and amplitude. [Article]. Journal of the Optical Society of America a-Optics Image Science and Vision, 28(6), 1297-1306.

This Article is brought to you for free and open access. It has been accepted for inclusion in Electrical and Computer Engineering Faculty Publications and Presentations by an authorized administrator of PDXScholar. Please contact us if we can make this document more accessible: pdxscholar@pdx.edu. 


\title{
Quantitative Carré differential interference contrast microscopy to assess phase and amplitude
}

\author{
Donald D. Duncan, ${ }^{1, *}$ David G. Fischer, ${ }^{2}$ Amanda Dayton, ${ }^{3}$ and Scott A. Prahl ${ }^{3}$ \\ ${ }^{1}$ Portland State University, 1900 SW 4th Avenue, Portland, Oregon 97201, USA \\ ${ }^{2}$ NASA Glenn Research Center, Cleveland, Ohio 44135, USA \\ ${ }^{3}$ Oregon Medical Laser Center, Providence St. Vincent Medical Center, Portland, Oregon 97225, USA \\ ${ }^{*}$ Corresponding author: donald.duncan@pdx.edu
}

Received February 24, 2011; revised April 22, 2011; accepted April 27, 2011;

posted April 27, 2011 (Doc. ID 143261); published May 31, 2011

\begin{abstract}
We present a method of using an unmodified differential interference contrast microscope to acquire quantitative information on scatter and absorption of thin tissue samples. A simple calibration process is discussed that uses a standard optical wedge. Subsequently, we present a phase-stepping procedure for acquiring phase gradient information exclusive of absorption effects. The procedure results in two-dimensional maps of the local angular (polar and azimuthal) ray deviation. We demonstrate the calibration process, discuss details of the phase-stepping algorithm, and present representative results for a porcine skin sample. ( 2011 Optical Society of America OCIS codes: $\quad 120.3180,120.5820,170.0180,170.3660,170.6935,180.3170$.
\end{abstract}

\section{INTRODUCTION}

It is evident that the first-order properties of light (color, scatter direction, polarization) can be used to infer properties of biological tissues with which it has interacted. Further, tissue structures and the instrumentation for acquiring local properties of the scattered light are often such that the spatial coherence length (a second-order property) of the illumination is on the order of the scale size of the inhomogeneity [1]. The coherence of the field affects its interaction with the medium, and in turn the coherence evolves in response to its interaction with the medium [2,3]. Thus, we are motivated to understand how coherence propagates within complex media.

There are two things required for an effective treatment of coherence propagation in biological tissues: first, a means of propagating the field through a complex, multiply scattering medium; second, a model that captures the structural properties of the medium. We have demonstrated that Monte Carlo techniques are capable of modeling diffraction and propagation of the field and its coherence function through free space $[\underline{4}, \underline{5}]$. What has been missing is a structured, second-order model of the propagation medium, i.e., biological tissue, that is compatible with the Monte Carlo formalism.

In a conventional treatment of propagation through a random medium, the medium is characterized in terms of a spatial power spectrum of the refractive index fluctuations [6]. This approach was followed by researchers studying propagation within highly scattering media, such as biological tissues. Schmitt and Kumar [7] used phase contrast microscopy of thin tissue sections and binarized these images prior to calculation of a spatial power spectrum. In acknowledging the conventional approach, they went so far as to refer to the "turbulent nature of tissues" and chose a characterization in terms of a von Kármán spectrum []. Others have since followed this lead $[\underline{9,10}]$.

In this paper, we choose a slightly different description. Instead of characterizing the medium in terms of its refractive index, we use the local ray deviation. Of course, this local ray deviation is proportional to the gradient of the phase, which in turn is the product of the wavenumber and optical path (physical path-index product). We chose this approach because of our interest in Monte Carlo modeling of propagation through strongly scattering media, and the fact that such studies characterize the medium properties in terms of a local ray deviation rather than a refractive index field. Moreover, there are a number of instruments for characterizing medium properties using phase gradient techniques. One such instrument is a differential interference contrast (DIC) microscope. In common use, DIC microscopes are used for a qualitative assessment of optically thin samples. A number of researchers, however, have developed schemes for deriving quantitative information from such imagery. For example, Preza et al. used DIC images at several azimuthal orientations to reconstruct phase [11]. Shribak and Inoué [12] recovered relative phase with a phasestepping approach, making use of the cosine relationship between the phase gradient and shear axis directions, and quasi-phase stepping using a precision rotation stage. In principle, this approach could determine absolute phase. In their study, however, the amount of shear was unknown, so only relative phase was recovered. Dana [13] performed a bias calibration and chose a bias setting about which the relationship between phase and intensity was approximately linear. Of course, this approach is limited to small phase excursions. In distinction to these studies is our interest in the direct measurable of the DIC microscope, i.e., the phase gradient.

The issue with DIC microscopy is that, to derive quantitative information, one must know the amount of image shear. Generally, however, microscope manufacturers do not make that information available. As a result, one must measure this parameter. Here again, other researchers have developed schemes for determining this. Mehta and Sheppard [14] directly measured shear by using an auxiliary Bertrand lens to inspect the wavefront interference in the back focal plane of the objective. Müller et al. [15] used a combination of 
fluorescence correlation spectroscopy and dynamic light scattering to determine shear to nanometer accuracy. Our accuracy demands are substantially less than this degree of precision.

There are other means of measuring the local scatter angle, notably that of Boustany et al. $[\underline{16}, \underline{17}]$. This is an interesting approach in which a series of images is acquired at differing numerical apertures. Differencing these images yields information on the polar scatter angle. An advantage of this technique is that it is insensitive to birefringence of the specimen. In fact, the birefringence could be assessed easily through complementary polarimetric measurements. Additionally, the azimuthal dependence of scatter can be assessed with more complex pupils synthesized using a spatial light modulator $[18,19]$. The drawback of this approach is complexity. Further, for discrimination of scatter that is highly forwardpeaked, this technique requires small numerical apertures.

An alternative approach that reconstructs absolute phase is that of through-focus imaging, or so-called transport of intensity DIC [20]. Here, the authors acquire a series of images at focus and on either side of focus and use the relationship between axial and transverse intensity gradients [21] to reconstruct the phase. This phase reconstruction was implemented with Fourier inversion of the Laplacian with regularization. The approach was demonstrated on an unstained tissue sample and validated using phase-stepping DIC; however, the sensitivity of the approach to absorption was not discussed.

Our specific objectives are to establish a simple means of calibrating a DIC microscope and to demonstrate the characterization of thin tissue samples in terms of the local ray deviation. From this characterization, one can subsequently develop first- and second-order models of the scatter within tissues. In this study, we use an unmodified commercial DIC microscope. We demonstrate a calibration procedure using a standard optical wedge, discuss details of a phase-stepping measurement technique, and show example measurement results on thin tissue sections.

\section{DIC MICROSCOPY}

In this section and the next, we provide some background on DIC microscopy and a particular phase-stepping method, the Carré four-step method, for retrieving quantitative information on local angular ray deviations across optically thin samples.

Shown in Fig. 1 is a conceptual diagram of a DIC microscope [22]. Such a microscope is typically operated in Köhler illumination mode, i.e., with the light source conjugate to the back focal plane of the condenser. Operation of the microscope can be understood by considering a single ray emanating from the (unpolarized, incoherent) source. Upon striking the first Nomarski prism, the ray is split into two orthogonally polarized components. The angular separation of the Nomarski and the condenser are matched such that the ray components striking the specimen are parallel. These rays are separated by a distance $s$, referred to as the image "shear." The polarizer is oriented such that the amplitudes of these components are equal. After passing through the objective, the orthogonally polarized ray components are physically superimposed by the upper Nomarski and then pass through the analyzer, which is oriented with its pass axis at $45^{\circ}$ with respect to the two components. The image shear is typically

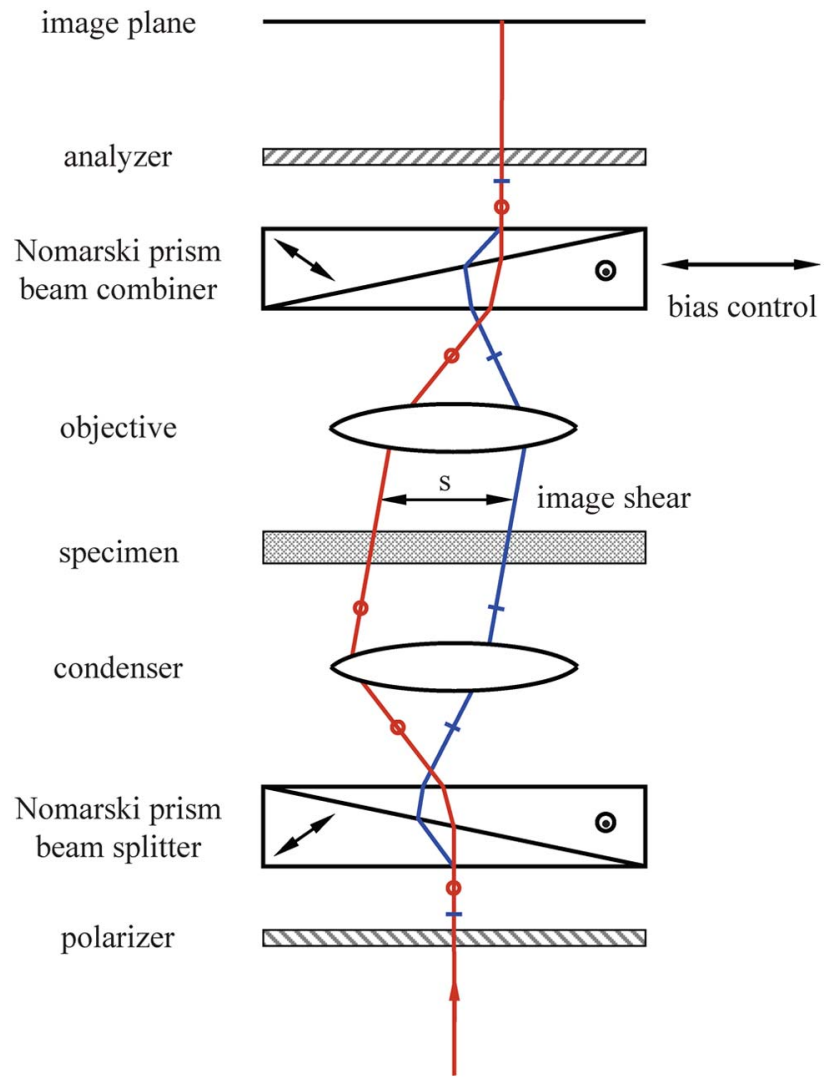

Fig. 1. (Color online) Illustration of the components of a Nomarski DIC microscope.

on the order of the microscope resolution, so the DIC microscope may be considered a common path interferometer [23].

The image produced by a DIC microscope may be expressed in the form [24]

$$
I(x, y)=A(x, y)\{1+\cos [\phi(x+s, y)-\phi(x, y)+\Psi]\},
$$

where $I$ is the measured intensity, $\phi$ is the object phase, and $\Psi$ is a phase offset that can be adjusted by changing the bias setting of the second Nomarski prism, and by inclusion of the term $A$, we have assumed a possible amplitude effect. In Eq. (1), it has also been assumed that the direction of shear (in the amount $s$ ) is in the $x$ direction. If the amount of shear is small compared to the microscope resolution, one can write

$$
I(x, y)=A(x, y)\{1+\cos [\Phi(x, y)+\Psi]\},
$$

where $\Phi(x, y)$ is the product of the shear and the phase gradient;

$$
\Phi(x, y)=s \frac{\partial \phi(x, y)}{\partial x}
$$

The local ray deflection in the $x$ direction can be recovered through the relationship [25]

$$
\sin \theta_{x}=\left[\frac{\Phi(x, y)}{k s}\right],
$$

where $k=2 \pi / \lambda$ is the free space wavenumber. For this calculation, one needs to know both $\Phi(x, y)$ and the amount of 
shear. The amount of shear, however, is generally not provided by the microscope manufacturer. Consequently, it must be determined through calibration, as outlined in Section 4 .

As shown above, Eq. (3) yields the local ray deflection in the $x$ direction (the direction of shear). However, to fully characterize the scatter from a sample, one must also determine the ray deflection, $\theta_{y}$, in the orthogonal direction. This is done by rotating the sample $90^{\circ}$ counter clockwise and repeating the measurement procedure, which we present in Section 3. Subsequent rotation of the resulting $\theta_{y}$ image clockwise by $90^{\circ}$ and registration with the first yields, for each pixel of the image, two corresponding scatter angles, $\theta_{x}$ and $\theta_{y}$. The actual polar and azimuthal scatter angles are derived according to the following formulas:

$$
\tan \eta=\sqrt{\tan ^{2} \theta_{x}+\tan ^{2} \theta_{y}}, \quad \tan \xi=\tan \theta_{y} / \tan \theta_{x}
$$

\section{CARRÉ FOUR-STEP METHOD}

The Carré four-step method [26] is one particular technique for determining the phase of an interference signal, such as the product of the phase gradient and the shear encoded in a DIC microscope image [see Eq. (2)]. The model for this procedure is

$$
I_{j}(x, y)=a(x, y)+b(x, y) \cos \left[\Phi(x, y)+\left(\frac{2 j-3}{2}\right) \beta(x, y)\right]
$$

where $j=0,1,2,3$ and the phase step $\beta(x, y)$ is generally unknown, but can be recovered by computing

$$
\tan \left[\frac{\beta(x, y)}{2}\right]=\sqrt{\frac{3\left[I_{1}(x, y)-I_{2}(x, y)\right]-\left[I_{0}(x, y)-I_{3}(x, y)\right]}{\left[I_{1}(x, y)-I_{2}(x, y)\right]+\left[I_{0}(x, y)-I_{3}(x, y)\right]}}
$$

Subsequently, the phase $\Phi(x, y)$ can be recovered from the expression

$$
\begin{aligned}
& \tan \Phi(x, y) \\
& =\tan \left[\frac{\hat{\beta}(x, y)}{2}\right] \frac{\left[I_{0}(x, y)-I_{3}(x, y)\right]+\left[I_{1}(x, y)-I_{2}(x, y)\right]}{\left[I_{1}(x, y)+I_{2}(x, y)\right]-\left[I_{0}(x, y)+I_{3}(x, y)\right]}
\end{aligned}
$$

Equation (5) is more general than the idealized expression of Eq. (1) in that it provides for an interference term that may not have unity visibility. We refer to the factor $b(x, y)$ as the modulation and the quotient $b(x, y) / a(x, y)$ as the visibility.

The hallmark of Carré methods is that the phase step $\beta$ need not be known a priori, and it may vary over the image field. As suggested by Eq. (6), there may be occasions when the quotient under the radical becomes negative. In this case, the phase-step angle is unknown. A convenient recourse, however, is to make use of a least-squares fit to the valid phasestep data points, i.e., ones for which the square root of Eq. (6) can be computed. Such an approach incorporates the $a$ priori knowledge that $\beta(x, y)$ varies slowly and continuously over the image field. After all, it is a property of the imaging apparatus and not the subject matter. This strategy is acknowledged explicitly in Eq. (7), where $\hat{\beta}(x, y)$ denotes the least-squares fit of the valid data points from Eq. (6) to a two-dimensional quadratic function.

Equations (6) and (7) provide estimates of the object phase and instrument phase step. However, an object also typically has amplitude variations, and this information is encoded in the mean image, $a(x, y)$, and the modulation, $b(x, y)$. An estimate of these two quantities is therefore desired (especially if one is modeling tissue). With the exception of various five, six, and seven-step Carré algorithms [27], there do not appear to be closed-form expressions for $a(\bar{x}, y)$ and $b(x, y)$. Moreover, existing formulas for these intensities have the same structure as Eq. (6), namely, square roots of combinations of measured images. In principle, these formulas are valid, but they break down in the presence of noise, which can render the term under the square root negative. A simple recourse is to seek the images that are most consistent with the previously established phase and phase-step estimates. We are thus led to the following set of simultaneous equations:

$$
\begin{aligned}
{\left[\begin{array}{c}
I_{0} \\
I_{1} \\
I_{2} \\
I_{3}
\end{array}\right] } & =\left[\begin{array}{ll}
1 & C_{0} \\
1 & C_{1} \\
1 & C_{2} \\
1 & C_{3}
\end{array}\right]\left[\begin{array}{l}
a \\
b
\end{array}\right] ; \\
C_{j} & =\cos \left[\Phi(x, y)+\left(\frac{2 j-3}{2}\right) \hat{\beta}(x, y)\right],
\end{aligned}
$$

where $\hat{\beta}(x, y)$ and $\Phi(x, y)$ have been computed respectively in Eqs. (6) and (7). Multiplying each side by the transpose of the "system" matrix, we get

$$
\left[\begin{array}{c}
\sum I_{j} \\
\sum C_{j} I_{j}
\end{array}\right]=\left[\begin{array}{cc}
4 & \sum C_{j} \\
\sum C_{j} & \sum C_{j}^{2}
\end{array}\right]\left[\begin{array}{l}
a \\
b
\end{array}\right]
$$

Note that (with the exception of the scalar 4) each term in Eq. (9) is a matrix of the same dimension, $M \times N$, of the original images. Least-squares solutions of this set of equations are provided by Cramer's rule:

$$
\begin{aligned}
\hat{a}(x, y) & =\frac{\sum I_{j} \sum C_{j}^{2}-\sum C_{j} I_{j} \sum C_{j}}{4 \sum C_{j}^{2}-\left(\sum C_{j}\right)^{2}}, \\
\hat{b}(x, y) & =\frac{4 \sum C_{j} I_{j}-\sum C_{j} \sum I_{j}}{4 \sum C_{j}^{2}-\left(\sum C_{j}\right)^{2}} .
\end{aligned}
$$

All of the above algebraic operations are performed point by point.

\section{MICROSCOPE CALIBRATION}

We have shown how ray deviation information is encoded into the phase of a DIC microscope image and how that phase can be recovered uniquely using the Carré four-step algorithm. To determine the local ray deviations themselves (in each of two orthogonal directions) across a sample, one must first estimate the amount of shear produced by the microscope, through calibration using an object of known phase gradient. A particularly simple phase object for this purpose is an optical wedge, depicted in Fig. 2 . 


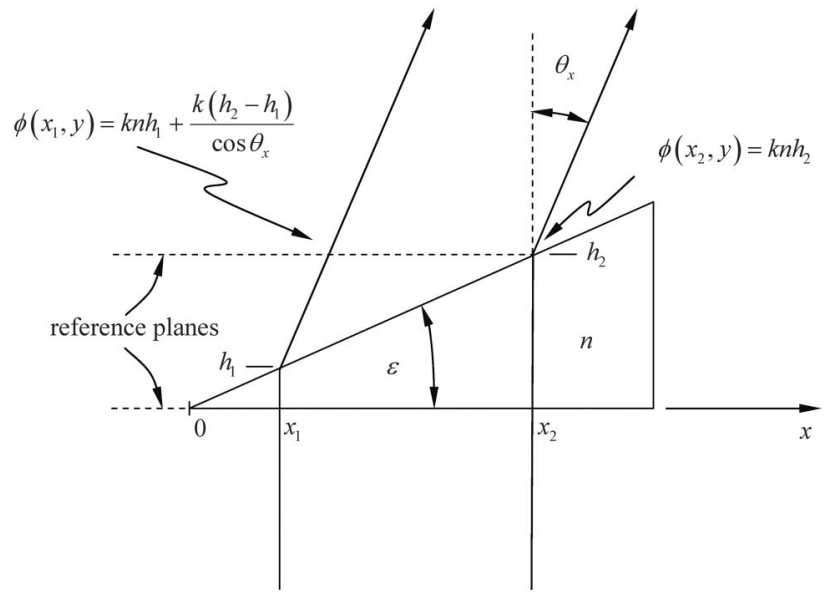

Fig. 2. Phases of rays deflected by an optical wedge.

As shown in Fig. 2, the phase difference between the two rays separated by a distance $s=x_{2}-x_{1}$ (the image shear) is

$$
\begin{aligned}
\phi\left(x_{2}, y\right)-\phi\left(x_{1}, y\right) & =\phi(x+s, y)-\phi(x, y) \\
& =k\left(h_{2}-h_{1}\right)\left(n-\frac{1}{\cos \theta_{x}}\right),
\end{aligned}
$$

where $n$ is the refractive index of the wedge and $h_{j}$ are the thicknesses at the positions $x_{j}$. Now recall that, in air, the deflection angle $\theta_{x}$ and the wedge angle $\varepsilon$ are related through the expression

$$
n \sin \varepsilon=\sin \left(\varepsilon+\theta_{x}\right) .
$$

Equations (11) and (12) can be combined to eliminate the deflection angle yielding an expression for the phase difference in terms of the physical properties of the wedge. For a wedge with small angular deviation, however, Eq. (11) can be written as

$$
\phi(x+s, y)-\phi(x, y)=k\left(h_{2}-h_{1}\right)(n-1) .
$$

Now consider Fig. 3 , which illustrates the orientation of the wedge angle with respect to that of the shear. With this angle defined as $\gamma-\gamma_{s}$, the thicknesses of the wedge at the two points $h_{1}$ and $h_{2}$ can be expressed as

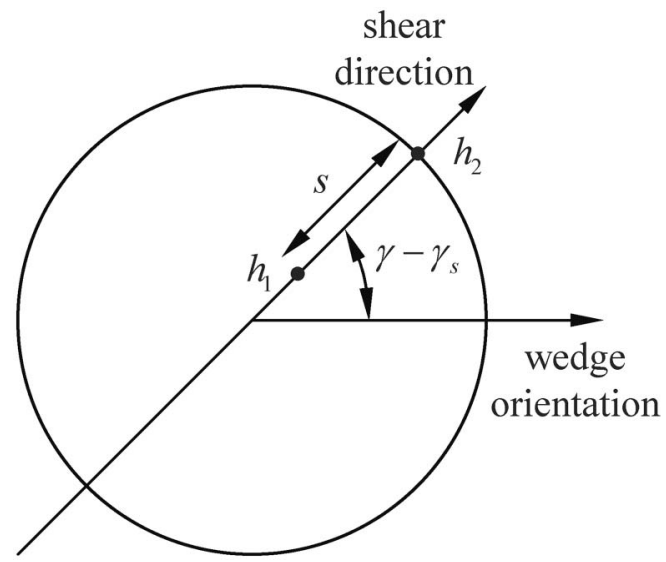

Fig. 3. Illustration of the effect of wedge orientation with respect to shear direction.
Table 1. Results of Global Fits to Data

\begin{tabular}{cccc}
\hline Parameter & R Channel & G Channel & B Channel \\
\hline$A$ & 94 & 87 & 87 \\
$\Phi_{0}$ & $70^{\circ}$ & $56^{\circ}$ & $46^{\circ}$ \\
$m$ & $118^{\circ}$ & $132^{\circ}$ & $142^{\circ}$ \\
$\gamma_{s}$ & $87^{\circ}$ & $87^{\circ}$ & $87^{\circ}$ \\
$\beta$ & $42^{\circ}$ & $47^{\circ}$ & $50^{\circ}$ \\
$s / \lambda$ & 2.0 & 2.2 & 2.4 \\
\hline \multicolumn{4}{c}{} \\
& $h_{2}=h_{1}+s \cos \left(\gamma-\gamma_{s}\right) \tan \varepsilon$.
\end{tabular}

It follows that the complete expression for the phase difference is

$$
\phi(x+s, y)-\phi(x, y)=k s(n-1) \tan \varepsilon \cos \left(\gamma-\gamma_{s}\right) .
$$

This result suggests that DIC measurements of a known wedge at a series of known azimuthal orientations can be used to estimate the shear distance $s$. In the following discussion, we present details of such a procedure.

We took a series of DIC images of a BK7 glass wedge with a purported $10^{\circ}$ ray deviation angle. The microscope to be calibrated was a Zeiss Axio Imager (transmission) microscope with an Epiplan NeoFluar HD DIC 10× NA 0.30 objective (Zeiss 44335). Condenser, objective, and diaphragms were adjusted to provide Köhler illumination. The light was linearly polarized and the condenser prism was a DIC I (Zeiss 426701). A $10 \times 0.30$ NA objective prism (Zeiss 444432) was used to adjust the bias. The translational position of the objective prism, and therefore the bias, was changed by turning a set screw in the housing of the prism. Thus, the phase relationship between the rays polarized parallel and perpendicular to the direction of shear was altered. Images were captured with a Nikon Digital Sight color camera that uses a Bayer filter. Exposure on the camera was manually set to $1 / 1000$ s. Each color channel was analyzed separately. All images were $960 \times 1280$ pixels with an object plane pixel size of $0.678 \mu \mathrm{m}$.

To evaluate the alignment of the condenser and objective prisms, a Bertrand lens was used to image the condenser and objective prism in the back focal plane of the objective. Each prism was imaged separately. With this imaging configuration, a dark line appeared perpendicular to the direction of shear. A least-squares fit of this line was performed and the slope of the fit was compared (1) as the set screw controlling the position of the objective prism was moved from its minimum to maximum location, (2) as the prism was manually shifted perpendicularly to the direction of shear, and (3) between the condenser and objective prisms. The angle of the line for five different rotations of the set screw changed less than $0.4^{\circ}$ degrees along its full extent of translation. This represents the maximum possible deviation between images. The objective prism was moved perpendicular to the direction of translation as much as possible within its housing and a maximum deviation of $0.3^{\circ}$ was found, thus demonstrating that the prism moves very little except in the direction of translation. The difference between the condenser and objective prism shear axes was $1.4 \pm 0.2^{\circ}$.

Measurement of the deflection of the wedge at $632.8 \mathrm{~nm}$ yielded a ray deviation angle of $9.79^{\circ}$; first surface reflection measurements yielded a wedge angle of $\varepsilon=17.8^{\circ}$. This angular deflection of the wedge is well within the $0.3 \mathrm{NA}\left(17.5^{\circ}\right)$ of 
the objective, so no vignetting was expected. With these wedge physical characteristics and considering the dispersion for BK7 [28] for the wavelengths of interest, $\cos \theta_{x} \approx 1$. As a result, the approximation of Eq. (13) is justified.

We chose a series of 13 wedge orientation angles at $\left(0^{\circ}, 15^{\circ}, 30^{\circ}, \cdots, 180^{\circ}\right)$, and for each orientation, captured images (at a fixed gain setting) for eight bias settings (corresponding to given number of turns of the bias knob). We employed two different procedures for estimating the shear distance $s$. The first was a global fitting procedure and the second was an estimation procedure based on the Carré algorithm.

\section{A. Global Fit}

The imaging model used for the global fit was

$$
I=A\left\{1+\cos \left[\Phi_{0}+m \cos \left(\gamma-\gamma_{s}\right)+N(\beta / 2)\right]\right\},
$$

where $N=0,1, \cdots, 7$ is the number of turns of the bias screw, and $\beta / 2$ is the step size (phase step per turn of bias screw of the upper Nomarski prism). This model represents a slight modification of Eq. (2), which introduces a fixed phase, $\Phi_{0}$. Each image was separated into three gray-level images for the red, green, and blue color channels. The mean value of each image was calculated. Thus, for the red channel, $12 \times$ $8=96$ values were used to determine the five values listed in Table 1 . The portion of the data at $\gamma=60^{\circ}$ was lost due to a file naming error. Results of the global fit are shown in Fig. 4.

From Eq. (15), we see that the constant $m$ is

$$
m=k s(n-1) \tan \varepsilon
$$

and, from the results of the global fit (Table 1), we arrive at the shear calibration factor for the red channel:

$$
\frac{s}{\lambda}=\frac{m}{2 \pi(n-1) \tan \varepsilon}=2.0
$$

where we used an assumed effective refractive index (considering the spectral weighting of the red color channel of the Bayer filter) of $n=1.5155$. Effective indices for the other color channels are $n=1.5182$ (green) and $n=1.5211$ (blue).

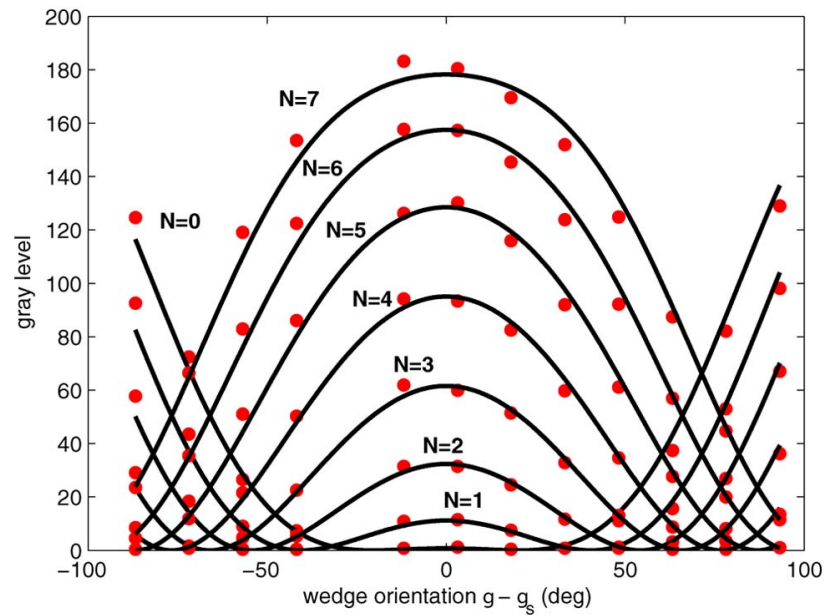

Fig. 4. (Color online) Global fit of data to Eq. (16) for red color channel. Numbers, $N$, refer to the number of turns of the bias screw on the upper Nomarski prism; the angle $\gamma-\gamma_{s}$ is the angular difference between the wedge orientation and shear direction.
Note that this estimate of image shear is consistent with those shown in [29]. The result $\gamma_{s}=87^{\circ}$ tells us that the shear axis was along the NW-SE direction and that there was a small error in the wedge orientation with respect to the shear axis of our microscope $\left(\sim 3^{\circ}\right)$. Variations with color channel in the estimate of this parameter are due simply to differing camera signal-to-noise ratios for the red, green, and blue colors. This angle is unimportant, however, if one has prior knowledge of the shear axis orientation.

\section{B. Carré Estimation}

Alternatively, these parameters can be determined using the Carré method. For each orientation of the wedge, we chose image sets corresponding to turns $(0,2,4,6)$ and $(1,3,5$, 7 ) of the bias screw. These sets were chosen because of requirements of the Carré algorithm; although Eqs. (ㅁ) and (7) are exact, noise considerations lead to a requirement on the total range of phases covered by stepping. The step size $\beta(x, y)$ was calculated for each pixel position using a set of four images. The simple mean of the image $\beta(x, y)$ is shown in Fig. $\underline{5}$ for the red color channel.

Each experiment corresponds to a set of four images (corresponding to turns $0,2,4,6$ or 1, 3, 5, 7 of the bias screw) for a particular orientation of the wedge. Two experiments were discarded because of an excessive number of pixels for which $\beta(x, y)$ could not be computed [see discussion of Eq. (6)]. The result shown here, $\beta=44^{\circ}$, is to be compared with that shown in Table $1\left(\beta=42^{\circ}\right)$. Results of the Carré algorithm for the phase, $\Phi$, are shown in Fig. $\underline{6}$. Here, too, the phases were averages computed over the entire image. Also shown in this figure is the fit model

$$
\Phi=\Phi_{i}+m \cos \left(\gamma-\gamma_{s}\right) .
$$

Parameters of this fit are

$$
m=120^{\circ}, \quad \Phi_{i}=129^{\circ} .
$$

The value of the slope parameter is to be compared with that derived from the global fit. To interpret the phase intercept in terms of the global fit we note that Eq. (ㅁ) can be written

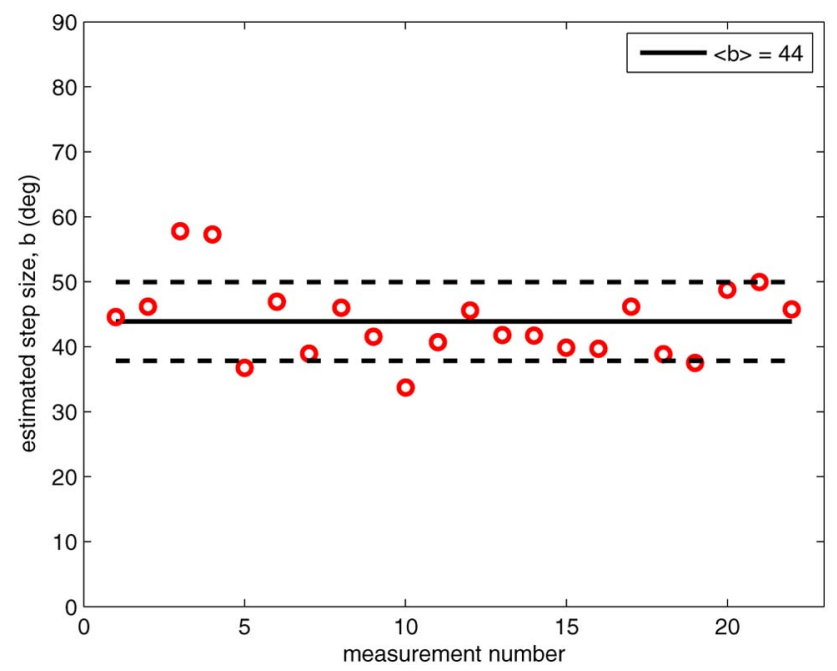

Fig. 5. (Color online) Step-size estimates from the Carré algorithm with mean, $\langle\beta\rangle$, plus or minus one standard deviation. 


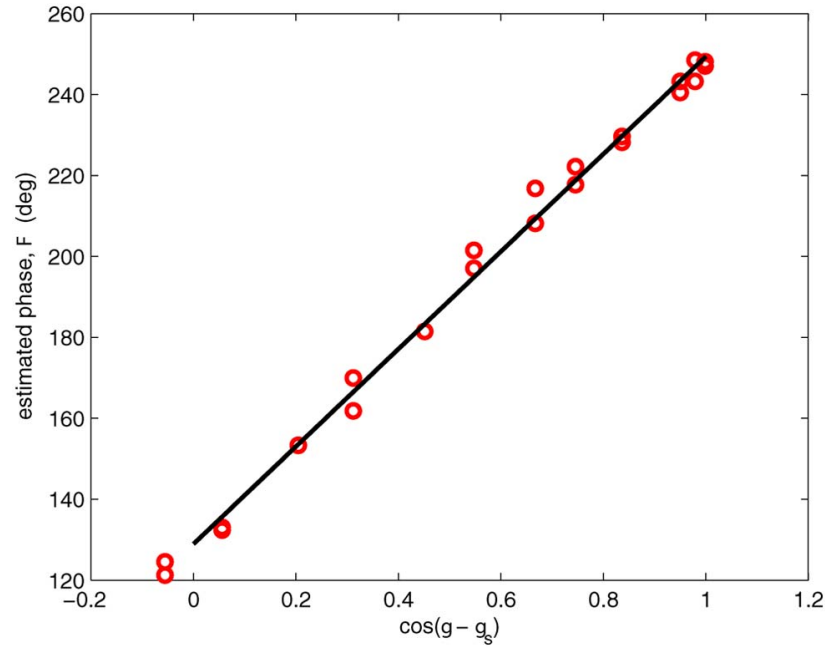

Fig. 6. (Color online) Carré-derived phases and fit to model. Fit model is $\Phi=\Phi_{i}+m \cos \left(\gamma-\gamma_{s}\right)$, where the slope and intercept values are respectively $m=120^{\circ}$ and $\Phi_{i}=129^{\circ}$.

$I_{j}(x, y)=a(x, y)+b(x, y) \cos \left[\Phi(x, y)-\left(\frac{3}{2}\right) \beta(x, y)+j \beta(x, y)\right]$

Thus,

$$
\Phi_{i}=129^{\circ}=\Phi_{0}+3 \beta / 2 \Rightarrow \Phi_{0}=63^{\circ},
$$

compared with the result from the global fit, $\Phi_{0}=70^{\circ}$. Values of the relevant model parameters derived from the Carré analyses for all three color channels are summarized in Table 2 . These values are to be compared with those in Table 1 .

Note that Born and Wolf [30] cite the resolution of a microscope with circular pupil and coherent illumination as

$$
\frac{\text { resolution }}{\lambda} \sim \frac{0.77}{\mathrm{NA}}=2.57 \text {. }
$$

Our finding that $s / \lambda \sim 2.0-2.4$ (depending on color channel) is consistent with this criterion and with the design strategy that one choose the largest shear possible in order to maximize sensitivity to phase gradients, but not large enough to manifest an image blur.

\section{TISSUE MEASUREMENTS}

The tissue sample employed for our study was obtained under an Institutional Animal Care and Use Committee-approved protocol. It was a biopsy of normal porcine skin that was fixed in formalin, embedded in paraffin, sectioned, and stained for Bromodeoxyuridine (BrdU), which replaces thymidine with uridine in the DNA of dividing cells.

Table 2. Parameter Values from Carré Analyses

\begin{tabular}{lccc}
\hline Parameter & R Channel & G Channel & B Channel \\
\hline$\Phi_{i}$ & $129^{\circ}$ & $123^{\circ}$ & $119^{\circ}$ \\
$\Phi_{0}=\Phi_{i}-3 \beta / 2$ & $63^{\circ}$ & $51^{\circ}$ & $42^{\circ}$ \\
$m$ & $120^{\circ}$ & $135^{\circ}$ & $145^{\circ}$ \\
$\beta$ & $44^{\circ}$ & $48^{\circ}$ & $52^{\circ}$ \\
$s / \lambda$ & 2.0 & 2.3 & 2.4 \\
\hline
\end{tabular}
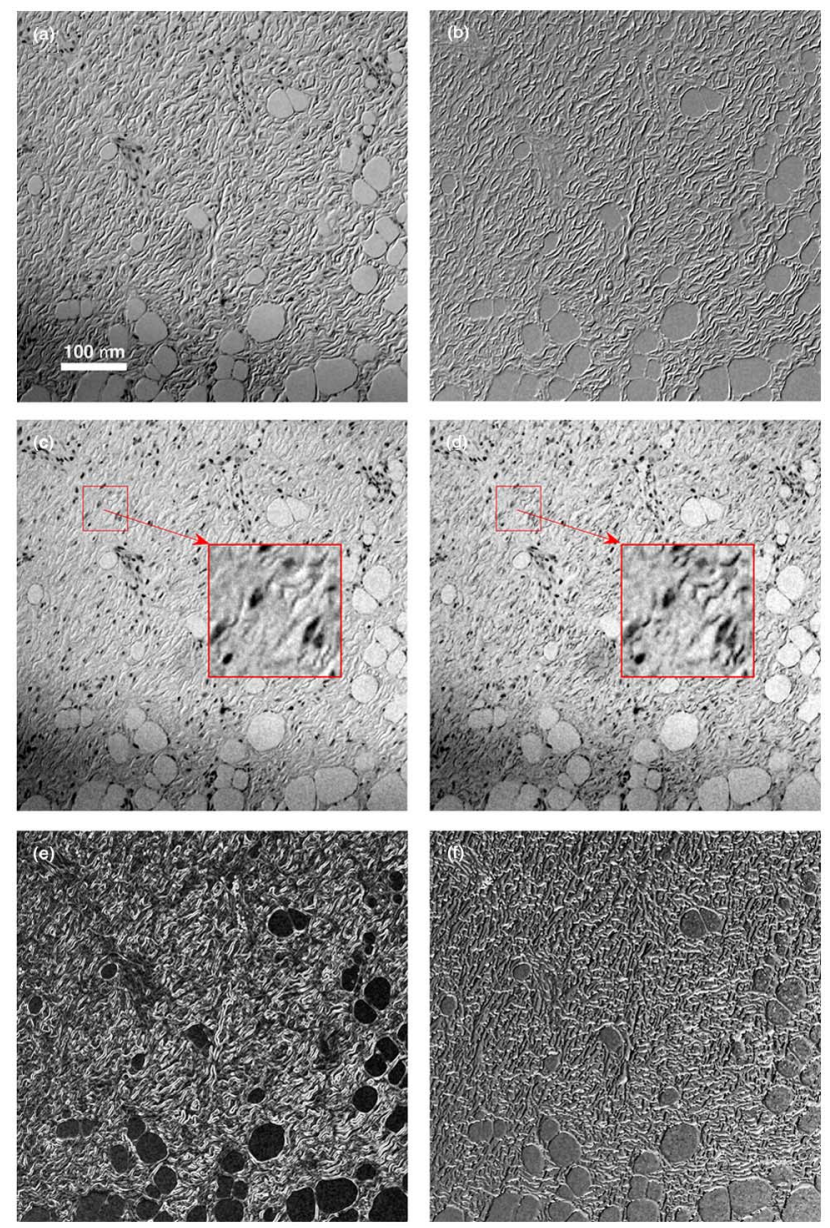

Fig. 7. (Color online) Montage of images illustrating processing of DIC data. (a) Original DIC image of porcine skin sample prepared with BrDU stain (red color channel); (b) grayscale encoded map of scatter angle, $\theta_{x}(x, y)$, along the axis of shear (range of angles displayed is $\left.\left(-2^{\circ}, 2^{\circ}\right)\right)$; (c) mean image, $a(x, y)$ [see Eq. (5)]; (d) modulation image, $b(x, y)$ [see Eq. (5)]; (e) grayscale-encoded map of polar scatter angle, $\eta(x, y)$ (range of angles displayed is $\left(0,2^{\circ}\right)$ ); (f) grayscale-encoded map of azimuthal scatter angle, $\xi(x, y)$ (range of angles displayed is $\left.\left(-180^{\circ}, 180^{\circ}\right)\right)$.

Shown in Fig. 7 is a montage of images illustrating the processing of the tissue data. Figure 7 (a) is the red color channel of the DIC image of porcine skin tissue prepared with BrDU stain. This is a view of the dermal tissue (epidermis is located beyond the top of the image). Globules at the bottom of the figure are subcutaneous fat.

For this tissue sample, a series of four microscope images were taken with bias knob settings of $(0,2,4,6)$. The pixel-bypixel values of the phase step $\beta(x, y)$ were computed via Eq. (6) and a quadratic surface fit (see Fig. 8) to the valid phase-step data estimates (valid fraction 0.995); Fig. 9 shows the probability density functions (PDFs) of the phase-step estimates and corresponding least-squares quadratic fit.

Figure $7(\mathrm{~b})$ is the grayscale encoded display of the scatter angle $\theta_{x}$ computed according to the formula

$$
\theta_{x}=\sin ^{-1}\left[\frac{\Phi-\Phi_{i}}{2 \pi(s / \lambda)}\right]
$$

where $\Phi_{i}=129^{\circ}$ and $s / \lambda=2.0$ (both of these parameter values are from the Carré analysis of the wedge calibration data). 


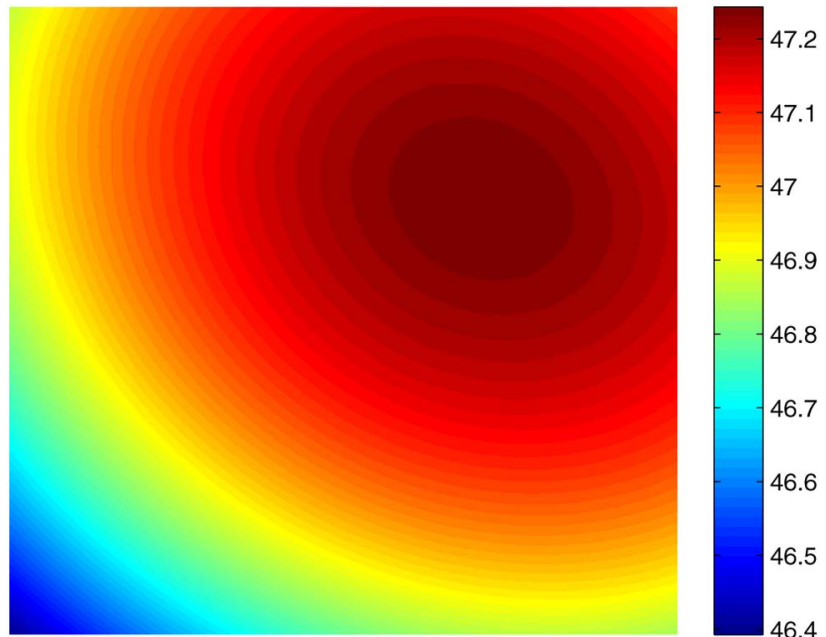

Fig. 8. (Color online) Quadratic least-squares fit to valid estimates of phase step, $\beta(x, y)$; units are degrees.

The mean and modulation images calculated according to Eq. (10) are displayed respectively in Figs. 7(c) and 7(d). Note that the mean image displays little, and the modulation image none, of the shadowing observed in the original DIC image. If one defines the modulation visibility as $v=b / a$, we expect to find, for a perfect system, that $v \equiv 1$. For this skin sample, we find the results shown in Fig. 10.

The tissue sample was rotated counterclockwise $90^{\circ}$ and a second phase-stepping analysis performed. The resulting $\theta_{y}$ map was rotated $90^{\circ}$ clockwise (in software) and registered with the previously derived $\theta_{x}$ map. This registration process began with a manual selection of six readily identifiable pointpairs in the two images and made use of a nonreflective similarity transformation, derived using a least-squares fit. Such transformations allow for scaling, translation, and any residual rotation not accounted for in the $90^{\circ}$ rotations. In deriving the registration transformation between these two data sets, the modulation images were used because they display less of the shadowing effect observed in the DIC imagery [see insets in Figs. 7(c) and 7(d)]. The final polar and azimuthal scatter angle maps were derived from the two registered

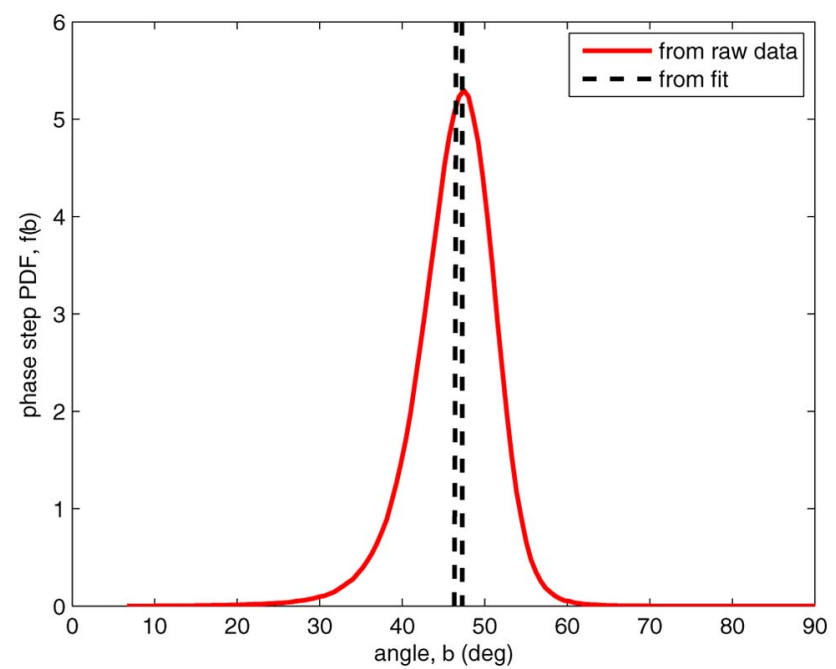

Fig. 9. (Color online) PDFs of valid phase-step angles and the corresponding fit shown in Fig. 8.

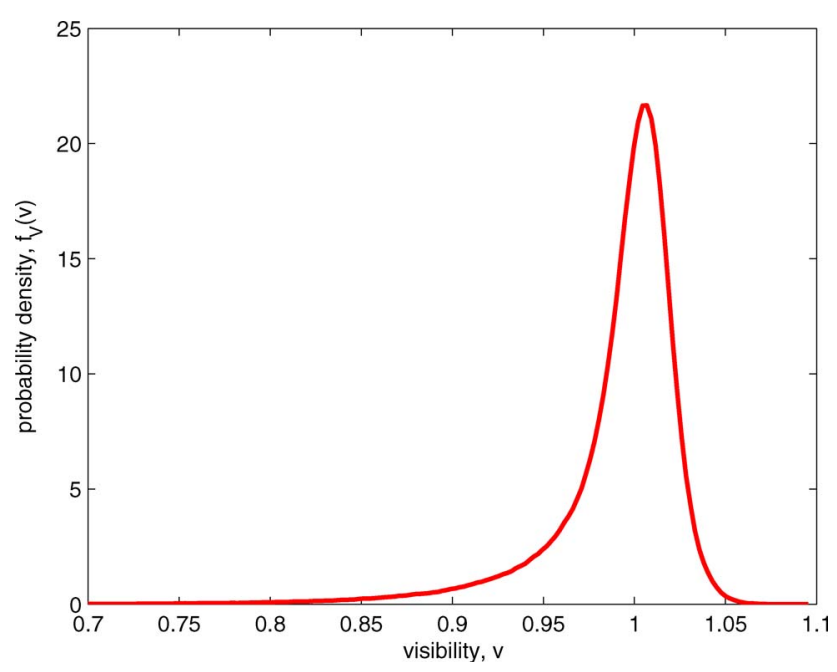

Fig. 10. (Color online) PDF of the modulation visibility for porcine skin sample.

ray deviation images according to Eq. (4). To date, we have not thoroughly evaluated the accuracy of this registration process or the impact of misregistration on the resulting scatter estimates. Figures $7(\mathrm{e})$ and $7(\mathrm{f})$ show respectively the polar, $\eta(x, y)$, and azimuthal, $\xi(x, \bar{y})$, ray deflection maps for the tissue sample. Each of these displays is a grayscale encoding of the angles in degrees. Figure 11 shows the first-order statistics. In the case of the polar scatter angle, we also display the best fit (based on peak value) Henyey-Greenstein (HG) phase function:

$$
P_{\mathrm{HG}}(\eta)=\frac{1}{2} \frac{1-g^{2}}{\left(1-2 g \cos \eta+g^{2}\right)^{3 / 2}} .
$$

The value of the asymmetry parameter, $g$, is a bit higher than expected for skin, as we discuss next.

The normalized light field (after passing through a sample with thickness $d$ and scattering coefficient $\mu_{s}$ ) is usually expressed in terms of unscattered and scattered parts:

$$
S(\cos \eta)=e^{-\mu_{s} d} \frac{1}{2 \pi} \delta(1-\cos \eta)+\left(1-e^{-\mu_{s} d}\right) P(\cos \eta),
$$

where the first term on the right-hand side represents the light that has passed through the sample without interacting (scattering) and the second term represents the scattered light. The scattering phase function is denoted $P(\cos \eta)$ and describes light that has been scattered at an angle of $\eta$ from the incoming direction. The scattered light is assumed to be azimuthally symmetric about the incoming direction. The zero and first moments of the scattering phase function are defined as

$$
\int_{4 \pi} P(\cos \eta) \mathrm{d} \Omega \equiv 1 \text { and } \int_{4 \pi} P(\cos \eta) \cos \eta \mathrm{d} \Omega \equiv g
$$

where $g$ is the scattering anisotropy and $d \Omega$ is the differential solid angle of integration.

Here, we define the full-field scattering anisotropy

$$
g^{\prime}=\int_{4 \pi} S(\cos \eta) \cos \eta \mathrm{d} \Omega
$$




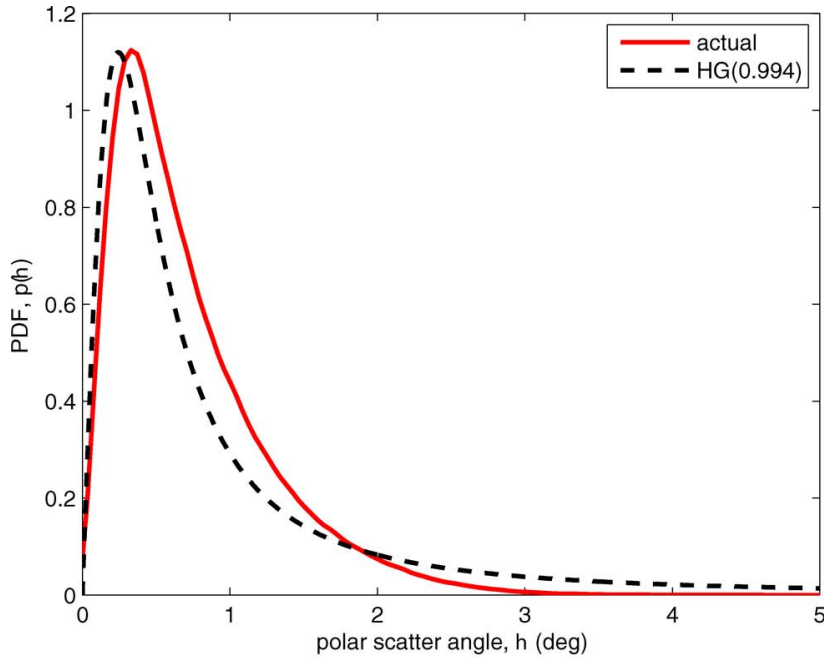

(a)

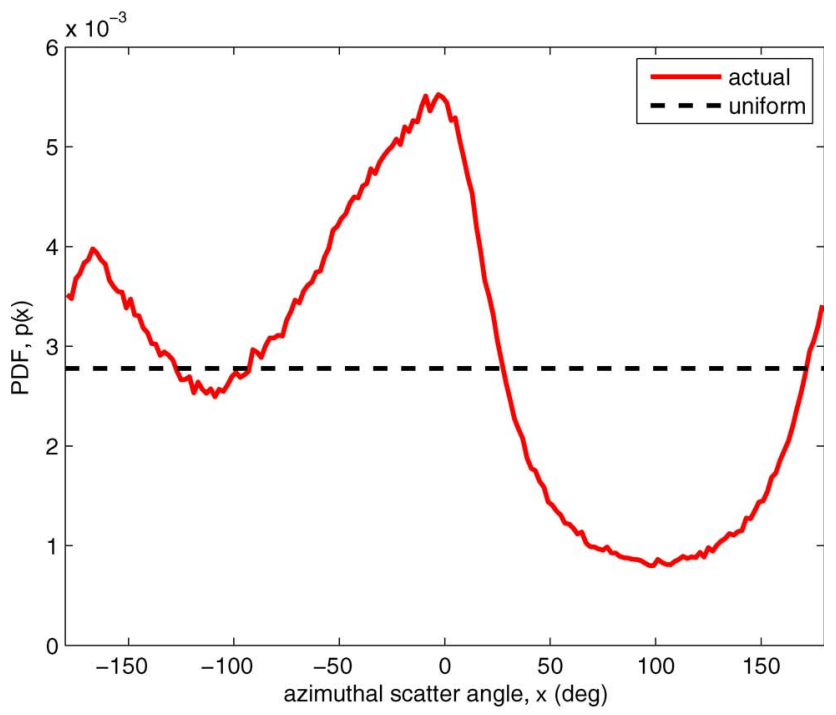

(b)

Fig. 11. (Color online) PDFs of polar (top) and azimuthal (bottom) scatter angles for tissue sample. Also shown for the polar scatter angle is the best approximate HG phase function. Note that the HG phase function has been multiplied by $\sin \eta$, thus giving the product the formal definition of a PDF. For the azimuthal angle, $\langle\cos \xi\rangle=0.148$, as opposed to $\langle\cos \xi\rangle=0$, for a completely uniform distribution.

$$
\begin{gathered}
g^{\prime}=e^{-\mu_{s} d} \int_{4 \pi} \frac{1}{2 \pi} \delta(1-\cos \eta) \cos \eta \mathrm{d} \Omega \\
+\left(1-e^{-\mu_{s} d}\right) \int_{4 \pi} P(\cos \eta) \cos \eta \mathrm{d} \Omega \\
g^{\prime}=e^{-\mu_{s} d}+\left(1-e^{-\mu_{s} d}\right) g .
\end{gathered}
$$

Our microscope slide sample was $\sim 5 \mu \mathrm{m}$ thick and typical values for skin are $\mu_{s} \approx 10 / \mathrm{mm}$ and $g=0.9$, therefore yielding an estimate for the full-field scattering anisotropy of

$$
g^{\prime}=0.95+0.05(0.9)=0.995 \text {. }
$$

Finally, we note that the HG phase function in Fig. 11 (top) has been multiplied by $\sin \eta$; this Jacobian factor is introduced naturally through the transformation of Eq. (4). The azimuthal statistic displays a near-uniform distribution with a slight

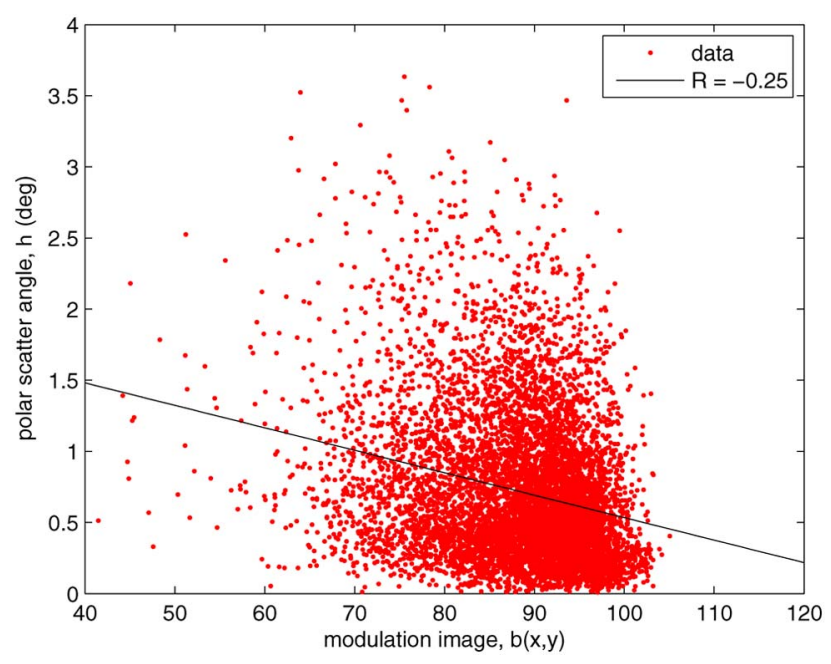

Fig. 12. (Color online) Relationship between polar scatter angle, $\eta(x, y)$, and grayscale values of the modulation image, $b(x, y)$. Line is least-squares fit; correlation is computed point by point.

preponderance of structure along the $\sim 0^{\circ}-180^{\circ}$ direction (NW to SE), which corresponds to the microscope shear axis.

The complete DIC processing algorithm yields spatially resolved maps of scatter angle and phase. To demonstrate that these maps possess distinct information, in Fig. $\underline{12}$, we show a scatterplot of the polar scatter angle, $\eta(x, y)$, versus the grayscale values of the modulation image values, $b(x, y)$. Also shown is the least-squares fit (the correlation was computed point by point). As this result demonstrates, the scatter angle is weakly correlated with the modulation image. Thus, in effect, we have separated the phase gradient and amplitude characteristics of the skin sample.

Finally, the second-order characteristic, in terms of the power spectral density (PSD), for the polar scatter angle is shown in Figs. 13 and 14. Figure 13 is a false color, logarithmic encoding of the two-dimensional PSD. Zero spatial frequency is at the center of this display and spatial frequency increases radially from this point. As seen in this figure, the PSD departs somewhat from rotational symmetry. This is simply a reflection of the fact that this tissue sample has structure with a preferred orientation. Thus, such a display is useful

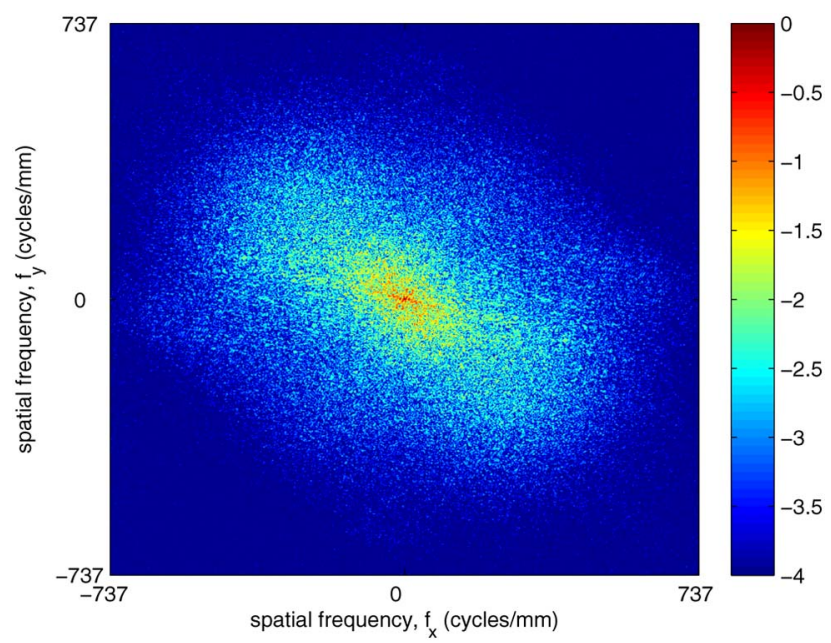

Fig. 13. (Color online) False color, $\log _{10}$, encoding of polar scatter angle PSD. DC is in center; axis limits are $\pm 1 / 2 p$, where $p$ is the pixel size $(0.678 \mu \mathrm{m})$. 


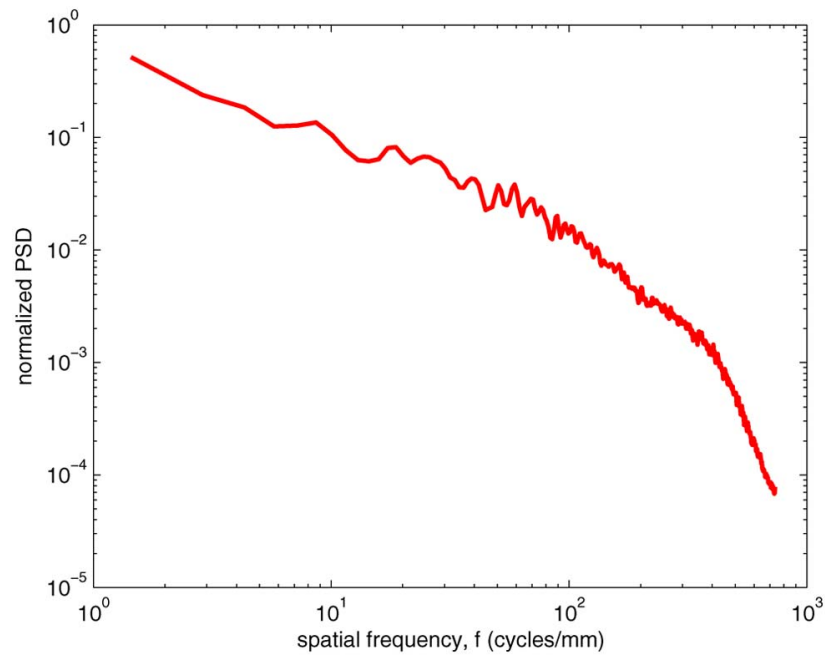

Fig. 14. (Color online) Azimuthally integrated polar scatter angle PSD.

for discerning organizational structure. Although the PSD is not isotropic, an azimuthal integration, shown in Fig. 14, provides some insight into the scale sizes of the scattering structures. Note that the PSD shown in Fig. 14 displays a number of distinct power law behaviors. These correspond to ranges of spatial structure sizes over which the tissue displays a self-similar scaling. Of course a complete analysis of these behaviors must take into account the DIC phase transfer function for the DIC microscope [31]. We note that the results shown in Figs. 13 and 14 are somewhat analogous to the von Kármán spectrum for refractive index, but are for the polar scatter angle rather than the refractive index. It is the scatter angle, rather than the phase, that is of more direct interest for Monte Carlo studies.

\section{DISCUSSION AND CONCLUSIONS}

We have demonstrated a technique whereby an unmodified DIC microscope can be used to provide quantitative estimates of the local ray deflection and wavefront of a field that has propagated through a thin tissue sample. The measurement concept relies on a calibration that we have described in detail. The essential feature of this calibration process is the use of a standard optical wedge placed on the microscope stage. The wedge is rotated through a series of known angles, thus generating a range of known phase gradients. Subsequent to the estimation of the shear using this procedure, we detailed a method of performing a Carré phase-stepping measurement to quantitatively assess thin tissue samples. This phase stepping was accomplished by means of a systematic positioning of the bias screw on the upper Nomarski prism. The end result of this procedure is a map of the azimuthal and polar scatter angles of the tissue sample. An outcome of this characterization is the ability to derive second-order statistics of the scatter caused by the tissue. Representative first- and second-order statistics were given for a thin section of porcine skin. First-order statistics were consistent with the HG model and the PSD of the polar scatter angle displayed a number of distinct power law dependencies.

A critical feature of the measurement concept described herein is the calibration of the DIC microscope. We presented a simple experimental technique using an optical wedge and two methods for analyzing the data obtained with this method.
Which of these two estimation procedures is the better to use is a matter for discussion, since both approaches use the data in quite different ways. On the one hand, the global fit makes use of all of the available data, because it is not constrained, as is the Carré, by the quotient of image intensities under the radical in Eq. (6) having to be nonnegative (although for this calibration, less than a few percent of the data were excluded on this basis). On the other hand, the Carré method (subject to the aforementioned constraint) provides a series of individual solutions for $\beta$ and $\phi$, upon which the estimate of $s$ is based. This distinction is somewhat mitigated by the fact that we use a fit, $\hat{\beta}$, for our estimate of the phase step. Nevertheless, it is the Carré phase stepping that we wished to use as the analysis method. As a matter of consistency, therefore, we chose to employ the calibration results derived from the Carré method. We believe that similarity of the calibration constants derived using the two approaches lends credence to use of the Carré method.

One possible concern with this calibration method was that the thickness of the optical wedge required the microscope stage to be lowered by about $6 \mathrm{~mm}$. This effectively moved the image plane further from the condenser prism than when a microscope slide was present, which may have affected the measured shear. For comparison, we used the fringe method recently described by Mehta and Sheppard [14], wherein the back focal plane of the objective is imaged with a Bertrand lens. When this technique was used to measure the shear of the DIC I objective prism alone, the shear was about $20 \%$ lower than that estimated using the wedge technique. This discrepancy might be due to the stage position (thereby increasing the shear in the image plane) or it may be a consequence of measuring only a single prism. Ultimately, we decided to use shear values obtained using the optical wedge because this configuration was closer to that used in the experiments (i.e., both prisms were present and data were collected in the image plane and not the pupil plane). In any case, the difference in shear values will only result in constant multiplicative correction for the phase or angle maps.

Kemao et al. [32] assessed the factors contributing to errors in phase estimation for the Carré four-step algorithm. They found that, for minimizing the effects of systematic and random intensity errors, a step size of $110^{\circ}$ was optimal. Their estimate of the optimal total phase-stepping range was thus approximately $330^{\circ}$. There are five- and seven-step Carré algorithms [27] that display optimal results for smaller phase steps, particularly these authors' A7 algorithm, which is optimal for a phase step of $54^{\circ}$. Note that, for the A7 algorithm, the total phase-stepping range is approximately $324^{\circ}$. From these examples, we see that the important factor in minimizing phase errors (due to intensity noise) is the total range for phase stepping; optimal is about $330^{\circ}$. The total phasestepping range for our microscope, however, is approximately $154-179^{\circ}$, depending on color channel. Another source of phase estimation error in Carré algorithms is due to errors in the phase stepping itself. Kemao et al. [32] addressed the effects of systematic (but not random) phase-step errors and found an optimal step size of $65.8^{\circ}$. Our microscope has a step size of $44-51^{\circ}$ depending on color channel. Novák et al. [27] also addressed this error source but did not cite an optimal step size for minimizing this effect. We estimate that the angular error in the positioning of the bias screw on the Nomarski prism is no more than $\pm 5^{\circ}$; the corresponding error 
in the phase step is thus $\pm 0.6^{\circ}$. At this point, it is unclear which noise source is dominant in our measurement procedure and, therefore, what the value of the optimal step size is. As a result, further study is required to fully assess the accuracy of the preliminary results presented herein. The technique used by Gutmann and Weber [33] may be useful in identifying the optimal step size as well as its estimation. Finally, error methods inherent in other methods of accomplishing the phase stepping, notably de Sénarmont [34], remain to be explored.

We note that, for DIC systems relying on birefringent optics to realize the image shear, our measurement concept is restricted to tissues displaying no birefringence. There is no such limitation for Köhler-DIC systems [31], for example.

While it is certainly possible to integrate two orthogonal measurements of the phase gradient to obtain the phase itself (to within an additive constant) [23], it is the phase gradient that is of greater practical interest. Such a characterization is compatible with Monte Carlo simulations of propagation, which make use of models of local ray deviation. Furthermore, a constant phase is associated with a homogeneous region, whereas the phase gradient is associated with structural variations. In addition to the application to numerical studies of propagation, the ability to characterize various tissues according to the PSD of the ray deflection may prove valuable in characterizing the scatter and absorption properties of tissues [1]].

\section{ACKNOWLEDGMENTS}

This work was sponsored in part by National Institutes of Health (NIH) grant NIH-NIDCR-R21-DE016758.

\section{REFERENCES}

1. H. Subramanian, P. Pradhan, Y. Liu, I. R. Capoglu, X. Li, J. D. Rogers, A. Heifetz, D. Kunte, H. K. Roy, A. Taflove, and V. Backman, "Optical methodology for detecting histologically unapparent nanoscale consequences of genetic alterations in biological cells,” Proc. Natl. Acad. Sci. USA 105, 20124-10129 (2008).

2. G. Gbur and E. Wolf, "Spreading of partially coherent beams in random media," J. Opt. Soc. Am. A 19, 1592-1598 (2002).

3. C. Mujat and A. Dogariu, "Statistics of partially coherent beams: a numerical analysis," J. Opt. Soc. Am. A 21, 1000-1003 (2004).

4. D. G. Fischer, S. A. Prahl, and D. D. Duncan, "Monte Carlo modeling of spatial coherence: free-space diffraction," J. Opt. Soc. Am. A 25, 2571-2581 (2008).

5. S. A. Prahl, D. G. Fischer, and D. D. Duncan, "A Monte Carlo Green's function formalism for the propagation of partially coherent light," J. Opt. Soc. Am. A 26, 1533-1543 (2009).

6. L. C. Andrews and R. L. Phillips, Laser Beam Propagation through Random Media (SPIE, 1998).

7. J. M. Schmitt and G. Kumar, "Turbulent nature of refractive index variations in biological tissue," Opt. Lett. 21, 1310-1312 (1996).

8. V. I. Tatarski, The Effects of the Turbulent Atmosphere on Wave Propagation (The National Oceanic and Atmospheric Administration, U. S. Department of Commerce and The National Science Foundation, 1971).

9. J. M. Schmitt and A. Knüttel, "Model of optical coherence tomography of heterogeneous tissue," J. Opt. Soc. Am. A 14, 12311242 (1997).

10. W. Gao, "Changes of polarization of light beams on propagation through tissue," Opt. Commun. 260, 749-754 (2006).

11. C. Preza, S. V. King, and C. J. Cogswell, "Algorithms for extracting true phase from rotationally-diverse and phase-shifted DIC images," Proc. SPIE 6090, 60900E (2006).

12. M. Shribak and S. Inoué, "Orientation-independent differential interference contrast microscopy,” Appl. Opt. 45, 460-469 (2006).
13. K. J. Dana, "Three dimensional reconstruction of the tectorial membrane: an image processing method using Nomarski differential interference contrast microscopy," Master's thesis (Massachusetts Institute of Technology, 1992).

14. S. B. Mehta and C. J. R. Sheppard, "Sample-less calibration of the differential interference contrast microscope," Appl. Opt. 49, 2954-2968 (2010).

15. C. B. Müller, K. Weiß, W. Richtering, A. Loman, and J. Enderlein, "Calibrating differential interference contrast microscopy with dual-focus fluorescence correlation spectroscopy," Opt. Express 16, 4322-4329 (2008).

16. N. N. Boustany, S. C. Kuo, and N. V. Thakor, "Optical scatter imaging: subcellular morphometry in situ with Fourier filtering," Opt. Lett. 26, 1063-1065 (2001).

17. N. N. Boustany, R. Drezek, and N. V. Thakor, "Calcium-induced alterations in mitochondrial morphology quantified in situ with optical scatter imaging,” Biophys. J. 83, 1691-1700 (2002).

18. J.-Y. Zheng, R. M. Pasternack, and N. N. Boustany, "Optical scatter imaging with a digital micromirror device," Opt. Express 17 20401-20414 (2009).

19. R. M. Pasternack, Z. Qian, J.-Y. Zheng, D. N. Metaxas, and N. N. Boustany, "Highly sensitive size discrimination of sub-micron objects using optical Fourier processing based on twodimensional Gabor filters," Opt. Express 17, 12001-12012 (2009).

20. S. S. Kou, L. Waller, G. Barbastathis, and C. J. R. Sheppard, "Transport-of-intensity approach to differential interference contrast (TI-DIC) microscopy for quantitative phase imaging," Opt. Lett. 35, 447-449 (2010).

21. M. R. Teague, "Deterministic phase retrieval: a Green's function solution,” J. Opt. Soc. Am. 73, 1434-1441 (1983).

22. D. Murphy, "Differential interference contrast (DIC) microscopy and modulation contrast microscopy," in Fundamentals of Light Microscopy and Digital Imaging (Wiley-Liss, 2001), pp. 153-168.

23. C. Preza, D. L. Snyder, and J.-A. Conchello, "Theoretical development and experimental evaluation of imaging models for differential-interference-contrast microscopy," J. Opt. Soc. Am. A 16, 2185-2199 (1999).

24. S. V. King, A. Libertun, R. Piestun, C. J. Cogswell, and C. Preza "Quantitative phase microscopy through differential interference imaging," J. Biomed. Opt. 13, 024020 (2008).

25. L. D. Landau and E. M. Lifshitz, The Classical Theory of Fields, 4th ed., Course of Theoretical Physics Series (Reed Elsevier, 2000), Volume 2.

26. K. Creath, "Phase-measurement interferometry techniques," in Progress in Optics, E. Wolf, ed. (Elsevier, 1988), Vol. 26, pp. 349-393.

27. J. Novák, P. Novák, and A. Mikš, "Multi-step phase-shifting algorithms insensitive to linear phase shift errors," Opt. Commun. 281, 5302-5309 (2008).

28. W. J. Tropf, M. E. Thomas, and T. J. Harris, "Properties of crystals and glasses," in Devices Measurements, \& Properties, 2nd ed., M. Bass, E. W. Van Stryland, D. R. Williams, and W. L. Wolfe, eds., Handbook of Optics (McGraw-Hill, 1995), Vol. II, pp. 33.3-33.101.

29. S. B. Mehta and C. J. R. Sheppard, "Quantitative phase retrieval in the partially coherent differential interference contrast (DIC) microscope," presented at Focus on Microscopy, Krakow, Poland, 5-8 April 2009.

30. M. Born and E. Wolf, Principles of Optics, 4th ed. (Pergamon, 1970).

31. S. B. Mehta and C. J. R. Sheppard, "Partially coherent image formation in differential interference contrast (DIC) microscope," Opt. Express 16, 19462-19479 (2008).

32. Q. Kemao, S. Fangjun, and W. Xiaoping, "Determination of the best phase step of the Carre algorithm in phase shifting interferometry," Meas. Sci. Technol. 11, 1220-1223 (2000).

33. B. Gutmann and H. Weber, "Phase-shifter calibration and error detection in phase-shifting applications: a new method," Appl. Opt. 37, 7624-7631 (1998).

34. S. Schwartz, D. B. Murphy, K. R. Spring, and M. W. Davidson, "de Sénarmont bias retardation in DIC microscopy," http://www .microscopyu.com/pdfs/DICMicroscopy.pdf (Nikon MicroscopyU, 2003). 